Artigo original

\title{
QUEIMA DE PASTAGENS EM RORAIMA, AMAZÔNIA BRASILEIRA: DINÂMICA DE CURTO PRAZO DOS NUTRIENTES NO SOLO
}

\author{
Reinaldo Imbrozio BARBOSA ${ }^{1}$ \\ Philip Martin FEARNSIDE ${ }^{2}$
}

\begin{abstract}
RESUMO: Foi estudada a dinâmica de curto prazo (< 1 ano) do teor de nutrientes (0-20cm) de uma pastagem estabelecida em um solo podzólico vermelho-amarelo (Ultisol) em Roraima, extremo norte da Amazônia brasileira. Os resultados indicaram que logo após a queima ( 7 dias), os teores absolutos de $\mathrm{Ca}^{+2}, \mathrm{Mg}^{+2}, \mathrm{~K}^{+}$, $\mathrm{PO}_{4^{-3}}$ e $\mathrm{S}$, juntamente com $\mathrm{O} \mathrm{pH}$, foram elevados. Na mesma fase, as concentrações de $\mathrm{Al}^{+3}, \mathrm{H}^{+}, \frac{\circ}{\mathrm{N}}$ (total) e \%MO sofreram uma redução no valor absoluto. Entretanto, em todos os casos, não houve diferença estatística significativa na concentração dos nutrientes nos períodos de curto prazo investigados. A elevação da fertilidade do solo com a queimada foi efêmera, recaindo a níveis não diferentes dos valores iniciais a partir de 120 dias após a queimada. Portanto, neste estudo de caso, há um indicativo de que a queima dos resíduos florestais e do capim não possui efeitos significativos na dinâmica da fertilidade do solo no curto prazo de pastagens derivadas de florestas.
\end{abstract}

Palavras-chave: Amazônia, nutrientes no solo, pastagens, Roraima

Pasture burning in Roraima, Brazilian Amazonia: short-term dynamics of soil nutrient content

ABSTRACT: The short-term (< 1 year) dynamics of soil nutrient content (0-20 cm) were studied in a pasture established on Ultisol in Roraima, northern Brazilian Amazonia. The results showed that just after burning (7 days), there had been increase, in absolute terms, in its levels $\mathrm{Ca}^{+2}, \mathrm{Mg}^{+2}, \mathrm{~K}^{+}, \mathrm{PO}_{4}^{-3}$ and $\mathrm{S}$, together with $\mathrm{pH}$. In this same phase, there were decreases, in absolute terms, in $\mathrm{Al}^{+3}, \mathrm{H}^{+}, \% \mathrm{~N}$ (total) and $\% \mathrm{OM}$. However, in all cases, no statistically significant difference in the concentrations of nutrients occurred over the short time periods studied. The increase in soil fertility was ephemeral, falling back to levels that not did differ from initial values after 120 days. Therefore, in this case study, there is an indicative that burns of both forest residues and grass no have significant effects in the short term dynamics of the soil fertility of pastures derived from forests.

Key-words: Amazonia, soil nutrients, Roraima, pastures

1 INPA/CPEC (Base de Roraima), R. Coronel Pinto 315 - Centro, Caixa Postal 96, 69301-970 Boa Vista Roraima, reinaldo@inpa.gov.br.

2 INPA/CPEC, Caixa Postal 478, 69011-970 Manaus - Amazonas, pmfearn@inpa.gov.br.

Bol. Mus. Integrado de Roraima, Boa Vista 6:9-16. 2000[2007] 


\section{INTRODUÇÃO}

Florestas tropicais convertidas para pastagens são o maior agroecossistema da região Amazônica (FEARNSIE, 1980 ; KAUFFMAN et al., 1998). O uso do fogo é comum nestes sistemas, não só como forma de manejo das ervas invasoras, como também por proporcionar maior vitalidade ao pasto, devido à transferência dos nutrientes da biomassa (resíduos florestais, ervas e capim) para o solo. A fertilidade e a conservação dos nutrientes em solos tropicais são reguladas, em parte pela quantidade de biomassa vegetal no sistema (JAIYBO \& MOORE, 1964 ; SCHUBART, 1977). Com as seguidas queimadas, aproximadamente cerca de $90 \%$ da biomassa remanescente dos resíduos florestais em sistemas de pastagens pode desaparecer em menos de 10 anos (BARBOSA \& FEARNSIDE, 1996). Com isto, o estoque de nutrientes fica quase que exclusivamente dependente da biomassa vegetal secundária e do capim do pasto. Este fato pode acarretar um declínio da fertilidade no longo prazo, levando a pastagem a se tornar improdutiva. Cronoseqüências de Iongo termo são importantes e vêm sendo investigadas em pastagens da Amazônia brasileira desde a década de 1970, no intuito de entender a fertilidade do solo como conseqüência da transformação de um sistema de alta, para outro de baixa biomassa (FALESI, 1976 ;

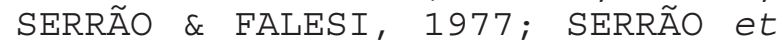
al., 1978). Entretanto, as medidas de curto prazo em agroecossistemas são pouco exploradas, embora sejam parte integrante da dinâmica de nutrientes do solo quando analisado o efeito instantâneo do fogo. O maior aporte de informações está estabelecido para o ato do desflorestamento seguido da queimada (ver SANCHEZ et al., 1983).
Este trabalho visou realizar um estudo de caso e acompanhar o comportamento dos teores de nutrientes no solo de uma pastagem na Amazônia, antes e, 7, 120 e 210 dias após uma queimada de "limpeza". A idéia geral era a de tentar entender o efeito do fogo sobre o processo de perda e recomposição nutricional (dinâmica) no curto prazo, neste tipo de sistema.

\section{DESCRIÇÃO DA ÁREA DE ESTUDO}

Nosso sítio de investigação estava localizado próximo à Vila do Apiaú $\left(02^{\circ} 33^{\prime} 11^{\prime \prime} \mathrm{N} ; 61^{\circ} 18^{\prime}\right.$ 27"W), que é a sede administrativa de uma área de colonização de mesmo nome, situada a $112 \mathrm{~km}$ a sudoeste da cidade de Boa Vista, Roraima, no extremo norte amazônico. O clima desta região é o "Ami" pelo sistema de classificação de Köppen e a média pluviométrica está situada entre 1900-2000 mm anuais (SANTOS et al., 1982; LAMEIRA \& COIMBRA, 1988). O pico do período chuvoso é entre maio e agosto e, o período seco está concentrado entre dezembro e março (BARBOSA, 1997). Exceto o Complexo da Serra de Mucajaí ( \pm $800 \mathrm{~m})$, o restante do relevo desta região está situado, em média, entre 120 e $180 \mathrm{~m}$.

O ambiente escolhido foi uma pastagem de 7 anos de idade com cobertura de Brachiaria humidicola (Rendle) Schweick. (Quicuio-daAmazônia), situada entre a vicinal 6 e a estrada tronco da colônia. Este sistema já havia sofrido três queimas anteriores (uma na conversão inicial e duas requeimas). O solo da área de estudos é o podzólico vermelhoamarelo (ultisol). Este tipo de solo ocupa a maior parte da região do Apiaú (Brasil, 1975) e pouco mais de 25\% de toda a Amazônia brasileira (MORAES et al., 1995). A vegetação original da área de experimentação era um confronto da Floresta Ombrófila Densa Sub- 
Queima de pastagens em Roraima...

montana (Ds) com outra de contato (Floresta Estacional / Ombrófila - ON), segundo as classes de vegetação definidas pelo IBGE (1992).

A pastagem foi queimada em fevereiro de 1993 (terceira requeima), aproveitando o controle e a limpeza das ervas invasoras que o proprietário do lote estava realizando como uma de suas atividades de trabalho daquele ano.

\section{AMOSTRAGEM DO SOLO}

As coletas neste sistema foram realizadas na camada superficial do solo $(0-20 \mathrm{~cm})$, aproveitando o desenho experimental de outro trabalho que estava sendo desenvolvido na área (ver BARBOSA, 1994). Este estudo se apoiava na distribuição de 36 sub-quadras distribuídas sob a forma de raios que partiam de dois pontos fixos dentro da pastagem. Estas subquadras estavam distribuídas em partes iguais tanto na pré, quanto na pós-queima.

Cada amostra foi composta por 3 sub-amostras correspondentes a uma sub-quadra. Na pastagem, foram coletadas 18 amostras na pré-queima e mais 18 aos 7 dias após a queima. Seqüencialmente, a pastagem foi amostrada aos $120(\mathrm{n}=12)$ e 210 ( $\mathrm{n}=12$ ) dias pós-queima. As coletas das duas últimas etapas foram realizadas sempre nas proximidades dos raios anteriormente montados.

A amostra-testemunha foi realizada na área de floresta adjacente à pastagem para que os resultados pudessem ser comparados. A coleta foi realizada em 12 amostras compostas ( 3 sub-amostras por ponto), divididas igualmente em cada lado da pastagem ( 6 no direito e 6 no esquerdo), sob a presunção de que a vegetação anterior era um contínuo sem alterações fisionômicas. Além disto, assumiu-se que os teores determinados na floresta não seriam alterados ao longo do tempo, presumindo estabilidade neste sistema.

\section{ANÁLISE DAS AMOSTRAS}

As amostras coletadas foram secas ao ar e, em seguida, foram realizadas as análises para os seguintes parâmetros: (1) pH (em água, método potenciométrico), (2) \% MO (matéria orgânica), por colorimetria, (3) \% N (nitrogênio total), por Kjeldahl, (4) $\mathrm{PO}_{4}^{-3}$ (fósforo assimilável), por colorimetria, (5) $\mathrm{Ca}^{+2}$ (cálcio) e $\mathrm{Mg}^{+2}$ (magnésio) trocável, ambos por espectrofotometria, (6) $\mathrm{K}^{+}$ (potásio trocável), por fotometria, (7) $\mathrm{Al}^{+3} \mathrm{e}+\mathrm{H}^{+}$(acidez trocável), por volumetria e (8) S (enxôfre), por turbidimetria. Todos os métodos de análise estão descritos por WILLIAMS \& STEINBERG (1959), EMBRAPA (1979) e VAN RAIJ $\&$ QUAGGIO (1983). Todos foram realizados no Centro de Energia Nuclear na Agricultura (CENA/USP) de Piracicaba - São Paulo.

Com o resultado laboratorial, foram determinados o desvio padrão e a existência de possíveis diferenças estatísticas entre as médias através da análise de variância (ANOVA ; 5\%).

\section{RESULTADOS E DISCUSSÃO}

A TABELA 1 apresenta as concentrações dos nutrientes determinados no solo da pastagem e da floresta ao longo das diferentes fases de amostragem. De modo geral, a pastagem pré-queima e os demais períodos do pós-queima apresentaram valores absolutos de teores de nutrientes e pH, qualitativamente superiores aos encontrados na floresta adjacente. Observação similar à de BUSCHBACHER et al. (1988) que analisaram 13 pastagens de diferentes idades, situadas no Pará, da mesma forma que outros autores como BRINKMANN \& NASCIMENTO (1973), DANTAS \& MATOS (1981), JORDAN et al. (1983) e HECHT (1982)

Bol. Mus. Integrado de Roraima, Boa Vista 6:9-16. 2000[2007] 
TABELA 1 - Concentração de nutrientes, matéria orgânica, carbono e pH no solo da floresta e da pastagem antes (pré-queima) e depois da queimada (pós-queima) nos tempos 7,120 e 210 dias.

\begin{tabular}{|c|c|c|c|c|c|c|c|c|c|c|c|}
\hline \multirow{2}{*}{$\begin{array}{c}\text { Tipo de } \\
\text { V egetação e } \\
\text { Etapa de } \\
\text { Coleta }\end{array}$} & \multirow[b]{2}{*}{$\begin{array}{l}\mathrm{n} \\
(\mathbf{1})\end{array}$} & \multirow[b]{2}{*}{$\begin{array}{c}\mathrm{pH} \\
(\mathrm{H} 2 \mathrm{O})\end{array}$} & \multirow[b]{2}{*}{$\begin{array}{l}\text { M O } \\
(\%)\end{array}$} & \multirow[b]{2}{*}{$\begin{array}{c}\mathrm{N} \\
(\%)\end{array}$} & \multicolumn{6}{|c|}{ N U T R I E N T E S (2) } & \multirow[b]{2}{*}{$\begin{array}{c}\mathrm{S} \\
\mathrm{ppm}\end{array}$} \\
\hline & & & & & $\mathrm{Al}^{+3}$ & $\mathrm{H}^{+}$ & $\begin{array}{l}\mathrm{Ca}^{+2} \\
(\mathrm{meq} /\end{array}$ & $\begin{array}{l}\mathrm{Mg}^{+2} \\
00 \mathrm{~g} \mathrm{de}^{-}\end{array}$ & $\begin{array}{c}\mathrm{K}^{+} \\
\text {solo) }\end{array}$ & $\mathrm{PO}_{4}^{-3}$ & \\
\hline FLOREST A & 12 & $\begin{array}{c}4.57 \\
(0.17)\end{array}$ & $\begin{array}{c}2.28 \\
(0.61)\end{array}$ & $\begin{array}{c}0.082 \\
(0.012)\end{array}$ & $\begin{array}{c}0.99 \\
(0.28)\end{array}$ & $\begin{array}{c}0.37 \\
(0.06)\end{array}$ & $\begin{array}{c}0.18 \\
(0.18)\end{array}$ & $\begin{array}{c}0.10 \\
(0.05)\end{array}$ & $\begin{array}{l}0.076 \\
(0.01)\end{array}$ & $\begin{array}{c}0.020 \\
(0.007)\end{array}$ & $\begin{array}{l}4.76 \\
(0.55)\end{array}$ \\
\hline $\begin{array}{l}\text { PAST AGEM } \\
\text {. pré-queima }\end{array}$ & 18 & $\begin{array}{c}5.29 \\
(0.52)\end{array}$ & $\begin{array}{l}3.06 \\
(0.54)\end{array}$ & $\begin{array}{c}0.072 \\
(0.012)\end{array}$ & $\begin{array}{c}0.46 \\
(0.21)\end{array}$ & $\begin{array}{c}0.27 \\
(0.09)\end{array}$ & $\begin{array}{c}0.73 \\
(0.95)\end{array}$ & $\begin{array}{c}0.26 \\
(0.25)\end{array}$ & $\begin{array}{l}0.074 \\
(0.05)\end{array}$ & $\begin{array}{c}0.043 \\
(0.042)\end{array}$ & $\begin{array}{c}5.54 \\
(0.48)\end{array}$ \\
\hline $\begin{array}{c}\text { pós-queima } \\
\text { (7 dias })\end{array}$ & 18 & $\begin{array}{c}5.68 \\
(0.53)\end{array}$ & $\begin{array}{c}2.68 \\
(0.53)\end{array}$ & $\begin{array}{c}0.068 \\
(0.010)\end{array}$ & $\begin{array}{c}0.31 \\
(0.21)\end{array}$ & $\begin{array}{c}0.21 \\
(0.06)\end{array}$ & $\begin{array}{c}1.03 \\
(0.70)\end{array}$ & $\begin{array}{c}0.32 \\
(0.20)\end{array}$ & $\begin{array}{l}0.080 \\
(0.03)\end{array}$ & $\begin{array}{c}0.055 \\
(0.036)\end{array}$ & $\begin{array}{c}5.88 \\
(1.93)\end{array}$ \\
\hline $\begin{array}{l}\text { pós-queima } \\
\text { (120 dias) }\end{array}$ & 12 & $\begin{array}{c}5.68 \\
(0.34)\end{array}$ & $\begin{array}{c}3.27 \\
(0.61)\end{array}$ & $\begin{array}{c}0.085 \\
(0.019)\end{array}$ & $\begin{array}{c}0.33 \\
(0.14)\end{array}$ & $\begin{array}{c}0.26 \\
(0.06)\end{array}$ & $\begin{array}{c}0.84 \\
(0.48)\end{array}$ & $\begin{array}{c}0.30 \\
(0.14)\end{array}$ & $\begin{array}{l}0.071 \\
(0.04)\end{array}$ & $\begin{array}{c}0.029 \\
(0.017)\end{array}$ & $\begin{array}{l}4.71 \\
(0.49)\end{array}$ \\
\hline $\begin{array}{l}\text { pós-queima } \\
(210 \text { dias })\end{array}$ & 12 & $\begin{array}{c}5.23 \\
(0.24)\end{array}$ & $\begin{array}{c}2.39 \\
(0.41)\end{array}$ & $\begin{array}{c}0.070 \\
(0.012)\end{array}$ & $\begin{array}{c}0.41 \\
(0.20)\end{array}$ & $\begin{array}{c}0.26 \\
(0.04)\end{array}$ & $\begin{array}{c}0.74 \\
(0.37)\end{array}$ & $\begin{array}{c}0.27 \\
(0.11)\end{array}$ & $\begin{array}{l}0.055 \\
(0.02)\end{array}$ & $\begin{array}{c}0.023 \\
(0.007)\end{array}$ & $\begin{array}{c}4.87 \\
(0.45)\end{array}$ \\
\hline
\end{tabular}

(1) núm ero de amostras compostas (3 sub-amostras cada uma).

(2) núm eros entre parenteses representam o desvio padrão.

que trabalharam em diferentes partes da Amazônia.

Os resultados de $\mathrm{pH}$ versus $\mathrm{Al}^{+3}$ e $\mathrm{H}^{+}$são normalmente conhecidos como dependentes entre si e correlacionados inversamente. $\mathrm{O} \mathrm{pH}$, que já se encontrava mais elevado na pré-queima da pastagem $(5,29)$ em relação à floresta adjacente $(4,57)$, alcançou níveis superiores 7 dias após a queimada $(5,68)$. Resultado semelhante foi determinado por MELO et al. (1994), em um desmatamento seguido de queima na região da Confiança III em Roraima (podzólico vermelhoamarelo). Com a queda da acidez, o alumínio e o hidrogênio trocável abaixaram suas concentrações. Nas amostragens da pós-queima de 210 dias (início da fase seca), os valores absolutos destes três parâmetros de análise mantiveram uma tendência de aproximação aos níveis iniciais da pastagem préqueima. Contudo, não foram visualizadas diferenças estatísticas significativas ao longo do período amostral e entre os dois ambientes avaliados (ANOVA $5 \%$ ).

A concentração média absoluta de cálcio (0,73 meq/100g de solo), magnésio $(0,26 \mathrm{meq} / 100 \mathrm{~g})$, potássio $(0,074 \mathrm{meq} / 100 \mathrm{~g})$, fósforo $(0,043$ $\mathrm{meq} / 100 \mathrm{~g})$ e enxôfre $(5,54 \mathrm{ppm})$ 
presentes na pastagem pré-queima, apresentaram uma tendência de elevação no pós-queima de 7 dias, devido ao grande aporte de cinzas e material particulado depositados sobre o solo. Este seria um fato esperado em queimadas na Amazônia (MARTINS et al., 1990). Entretanto, da mesma forma que o pH, não ficaram evidenciadas diferenças significativas entre os valores observados no nível de 5\% (ANOVA). SMYTH \& BASTOS (1984) também relataram que não havia significância nos valores dos teores de nutrientes antes e depois de uma queimada de Pueraria phaseoloides em um latosolo amarelo álico, próximo de Manaus. Isto pode sugerir que a baixa quantidade de biomassa presente nos sistemas de pastagens não elevaria os teores de nutrientes em níveis significativos.

Dos elementos químicos acima citados, apenas o valor absoluto do fósforo assimilável da pastagem no pós-queima de 210 dias $(0,023$ meq/100g) chamou a atenção devido à redução quase pela metade do valor encontrado na pré-queima $(0,043$ meq/loog de solo). Este mesmo comportamento do fósforo em pastagens originadas de floresta tropical foi observado por BUSCHBACHER ( 1984 ) em um experimento em San Carlos (Venezuela), sendo um fato comum neste tipo de situação (KAUFFMAN et al., 1992).

O nitrogênio $(0,072 \%$ e a matéria orgânica $(3,06 \%)$ presentes no solo da pastagem pré-queima foram reduzidos imediatamente após a queimada, e tiveram um comportamento semelhante ao longo das medidas de curta duração observadas. Na seqüência, ambos sofreram uma elevação absoluta na fase mais chuvosa da amostragem (pós-queima 120 dias), decaindo com o início do período seco na região (pós-queima 210 dias). A matéria orgânica do solo está intimamente ligada às condições edáficas que afetam a decomposição da biomassa nas camadas do solo. A conversão de florestas em pastagens reduz a capacidade de estoque de água do solo e pode confinar a entrada de carbono apenas pela biomassa das raízes das gramíneas situadas nas camadas superiores (CHAUVEL et al., 1991; NEPSTAD et al., 1994). Portanto, neste caso específico, é mais provável que as condições edáficas ao longo do período analisado tenham contribuído mais do que o efeito do fogo no que se refere à dinâmica da concentração de MO no solo.

\section{CONCLUSÕES}

Nossas medidas de curto prazo indicaram que, embora haja uma melhora qualitativa (absoluta) nos teores de nutrientes e de pH logo após a queimada, estes não são significativos e não se sustentam ao longo de um espaço inferior a 4 meses, retornando a níveis muito próximos dos estabelecidos antes do efeito do fogo. A baixa idade do sistema investigado ( 7 anos) pode ter afetado este resultado. Neste estágio, o sistema ainda possui um aporte de massa orgânica remanescente que cobre parcialmente os custos nutricionais do ambiente, diferente de pastagens acima de 10 anos e estabelecidas em solos argilosos que se degradam rapidamente como sugerido por SERRÃO \& FALESI (1977). Além disto, a densidade do solo não foi utilizada neste estudo como fator de correção dos estoques de nutrientes tanto da pastagem como da floresta adjacente. Entretanto, a idéia geral do estudo foi a de analisar o efeito do fogo na concentração de nutrientes no curto prazo, e não o efeito da troca do uso da terra. Por fim, a natureza efêmera dos aumentos da fertilidade do solo neste estudo de caso de Roraima, indica que a queima dos resíduos florestais e do capim não possui efeitos significativos na

Bol. Mus. Integrado de Roraima, Boa Vista 6:9-16. 2000[2007] 
dinâmica da fertilidade do solo no curto prazo de pastagens derivadas de florestas.

\section{AGRADECIMENTOS}

A Empresa Brasileira de Pesquisa Agropecuária de Roraima ( EMBRAPA-RR) cedeu seus laboratórios para a preparação inicial das amostras de solo. O Laboratório de Fertilidade do Solo do Centro de Energia Nuclear na Agricultura (CENA/USP) realizou as análises de nutrientes. A Fundação Banco do Brasil (FBB n 10/1615-2) e o Pew scholars Program in Conservation and the Environment financiaram parte do trabalho.

\section{BIBLIOGRAFIA CITADA}

Barbosa, R.I. 1994. Efeito Estufa na Amazônia: Estimativa da Biomassa e a Quantificação do Estoque e Liberação de Carbono na Queima de Pastagens Convertidas de Florestas Tropicais em Roraima, Brasil. Dissertação de Mestrado. INPA/UFAM, Manaus. 85p.

Barbosa, R.I. 1997. Distribuição das Chuvas em Roraima. In: Barbosa, R.I.; Ferreira, E.J.G.; Castellon, E.B. (eds.), Homem, Ambiente e Ecologia no Estado de Roraima. INPA, Manaus. p. 325-335.

Barbosa, R.I.; Fearnside, P.M. 1996. Pasture burning in Amazonia: dynamics of residual biomass and the storage and release of aboveground carbon. Journal of Geophysical Research (Atmospheres), 101 (D20): 25.847-25.857.

Brasil 1975. Projeto RADAMBRASIL. Levantamento de Recursos Naturais (vol. 8). Ministério das Minas e Energia. Rio de Janeiro. 428p.

Brinkmann, W.L.F.; Nascimento, J.C. 1973. The effect of slash and burn agriculture on plant nutrients in the Tertiary region of Central Amazonia. Acta Amazonica, 3(1) : 5561 .
Buschbacher, R. 1984. Changes in Productivity and Nutrient Cycling Following Conversion of Amazon Rainforest to Pasture. PhD Thesis, University of Georgia, Athens, Georgia. 193p.

Buschbacher, R.; Uhl, C.; Serrão, A.S. 1988. Abandoned pastures in eastern Amazonia. II . Nutrient stocks in the soil and vegetation. Journal of Ecology, 76: 682-699.

Chauvel, A.; Grimaldi, M.; Tesseir, D. 1991. Change in pore-space distribution following deforestation and revegetation: and example from the Central Amazon Basin, Brazil. Forest Ecology and Management, 38: 247-257.

Dantas, M.; Matos, A.O. 1981. Estudos fito-ecológicos do trópico úmido brasileiro. III. Conteúdo de nutrientes em cinzas de floresta e capoeira, Capitão Poço-PA. Boletim de Pesquisa, 14 (EMBRAPA/CPATU Belém) . 23p.

EMBRAPA 1979. Manual de Métodos de Análise de Solo. EMBRAPA / SNLCS. Rio de Janeiro.

Falesi, I.C. 1976. Ecossistema de pastagem cultivada na Amazônia Brasileira. Boletim Técnico, 1 (EMBRAPA/CPATU - Belém). 193 p.

Fearnside, P.M. 1980. The effects of cattle pasture on soil fertility in the Brazilian Amazon: consequences for beef production sustainability. Tropical Ecology, $21(1): 125-137$.

Hecht, S.B. 1982. Cattle Ranching Development in the Eastern Amazon: Evaluation of a Development Policy. PhD Thesis. University of California. 454p.

IBGE 1992. Manual Técnico da Vegetação Brasileira. Manuais Técnicos em Geociências $n^{\circ}$ l. IBGE, Rio de Janeiro. 92p.

Jaiybo, E.O.; Moore, A.W. 1964. Soil fertility and nutrient storage in different soil-vegetation systems in a tropical rain forest

BARBOSA, R.I. \& FEARNSIDE, P.M. 
Queima de pastagens em Roraima...

environmental.

Agriculture, 41(2): 129-139.

Jordan, C.; Caskey, W.; Escalante, G.; Herrera, R.; Montagnini, F.; Todd, R.; Uhl, C. 1983. Nitrogen dynamics during conversion of primary Amazonian Rain Forest to slash and burn agriculture. Oikos, 40: 131139 .

Kauffman, J.B.; Till, K.M.; Shea, R.W. 1992 . Biogeochemistry of deforestation and biomass burning. In: Dunnette, D.A.; Obrien, R.J. (eds.), The Science of Global Change: The Impact of Human Activities on the Environment. American Chemical Society Symposium Series n. 483. American Chemical Society, Washington D.C. p. 426450 .

Kauffman, J.B.; Cummings, D.L. ; Ward, D.E.; Babbitt, R. 1998. Fire in the Brazilian Amazon: 2. Biomass, nutrient pools and losses in cattle pastures. Oecologia, 113(3) : 415427 .

Lameira, O.A.; Coimbra, J.M. 1988. Levantamento e Distribuição da Precipitação em Roraima. Empresa Brasileira de Pesquisa Agropecuária - Unidade Experimental de Âmbito Territorial (EMBRAPA/UEPAT) de Boa Vista - Roraima. Pesquisa em Andamento, 12. Boa Vista. 5p.

Martins, P.F.S.; Cerri, C.C.; Volkoff, B.; Andreux, F. 1990. Efeito do desmatamento e do cultivo sobre características físicas e químicas do solo sob floresta natural na Amazônia Oriental. Revista do Instituto de Geociências (São Paulo), 11(1): 21-33.

Melo, G.W.B.; El-Husny, J.C.; Coelho, M.E.P. 1994. Alterações nas características químicas de um solo sob floresta tropical após a queima da vegetação. Pesquisa em Andamento, 2 (EMBRAPA-CPAF/RR). $2 \mathrm{p}$.

Moraes, J.L.; Cerri, C.C.; Melillo, J.M. ; Kicklighther, D.; Neill, C.; Skole, D.L.; Steudler, P.A. 1995. Soil carbon stocks of the Brazilian
Amazon basin. Soil Science Society of America Journal, 59(1): 244247 .

Nepstad, D.; Carvalho, C.R.; Davidson, E.A.; Jipp, P.H.; Lefebvre, P.A.; Negreiros, G.H.; Silva, E.D.; Stone, T.A.; Trumbore, S.E.; Vieira, S. 1994. The role of deep roots in the hydrological cycles of Amazonian forests and pastures. Nature, 372: 666-669.

Sanchez, P.A.; Villachica, J.H. ; Bandy, D.E. 1983. Soil fertility dynamics after clearing a tropical rainforest in Peru. Soil Science Society of America Journal, 47(6): $1171-1178$.

Santos, R.D.; Gama, J.N.R.F.; Soares, A.F. 1982 . Levantamento de reconhecimento de baixa intensidade dos solos e avaliação da aptidão agrícola das terras do projeto de colonização Apiaú, Território Federal de Roraima. Boletim de Pesquisa, 14 (EMBRAPA/SNLCS) . 175p.

Schubart, O.H. 1977. Critérios Ecológicos para o Desenvolvimento Agrícola das Terras Firmes da Amazônia. CNPq/INPA, Manaus. 29p.

Serrão, E.A.S.; Falesi, I.C. 1977. Pastagens do Trópico Úmido Brasileiro. EMBRAPA / CPATU, Belém. $63 \mathrm{p}$.

Serrão, E.A.S.; Falesi, I.C.; Veiga, J.B.; Neto, J.F.T. 1978 . Produtividade de Pastagens Cultivadas em Solos de Baixa Fertilidade das Áreas de Floresta do Trópico Úmido Brasileiro. Belém, EMBRAPA/CPATU. 73p.

Smyth, T.J.; Bastos, J.B. 1984. Alterações na fertilidade de um latossolo amarelo álico pela queima da vegetação. Revista Brasileira de Ciência do Solo, 8: 127-132.

van Raij, B.; Quaggio, J.A. 1983. Métodos de análise de solo para fins de fertilidade. Boletim Técnico, 81 (IAC/SP - Campinas). $31 \mathrm{p}$. 
Williams, C.H.; Steinberg, A. 1959.

Soil sulphur fractions as chemical indices of avaliable sulphur in some Australian soils. Australian Journal of Agricultural Science, $10(2): 340-352$. 